DOI: 10.12957/demetra.2017.22407

\title{
Adesão às orientações nutricionais: uma revisão de literatura
}

\section{Adherence to nutritional orientations: a literature review}

\author{
Kelly Cristina Araujo Estrela \\ Anna Carolina Di Creddo Alves' \\ Tatiana Teixeira Gomes' \\ Mistue Isosaki ${ }^{1}$ \\ 1 Universidade de São Paulo, Instituto do \\ Coração do Hospital das Clínicas da Faculdade \\ de Medicina, Serviço de Nutrição e Dietética. São \\ Paulo-SP, Brasil. \\ Correspondência / Correspondence \\ Kelly Cristina Araujo Estrela \\ kellycristinaae@gmail.com
}

\section{Resumo}

A adesão dos pacientes às orientações nutricionais é imprescindível para que se obtenham os resultados esperados da educação alimentar. O fenômeno da adesão e não adesão traz grande impacto na morbimortalidade de indivíduos que são acometidos por doenças crônicas e é um dos problemas mais importantes enfrentados pelos profissionais de saúde. Este trabalho teve como objetivo identificar como é avaliada a adesão às orientações nutricionais e seus determinantes, além de sumarizar formas para melhorar a adesão. Realizou-se uma revisão de literatura nas bases de dados eletrônicas SciELO, LILACS, PubMed e ClinicalKey, no período de 2005 a 2015. Ao longo da revisão literária, constatouse que a avaliação da adesão pode ser subjetiva ou objetiva e que é feita com diferentes tipos de ferramentas por causa da grande dificuldade encontrada na maneira de mensurar essa variável. Várias interfaces influenciam a adesão ao tratamento, como o paciente, a doença, a terapia, os aspectos socioeconômicos e culturais, o sistema de saúde e o relacionamento com os profissionais de saúde. Devido à complexidade da dimensão da temática adesão, não existe um método ou instrumento que se adapte a todos os objetivos e análises. Contudo, são necessários estudos metodológicos que elaborem e validem instrumentos de aferição da adesão às orientações nutricionais.

Palavras-chave: Adesão do Paciente. Terapia Nutricional. Orientação. Avaliação. Fidelidade a Diretrizes. 


\section{Abstract}

Patient compliance with nutritional orientations is essential to achieve the expected results of nutritional education. The phenomenon of adherence and non-adherence brings great impact on morbidity and mortality in individuals affected by chronic diseases, and it is one of the major problems faced by health professionals. The aims of this study were to identify how adherence to nutritional orientations and its determinants are assessed, and to summarize ways to improve adherence. A literature review has been made in the electronic databases SciELO, LILACS, PubMed and ClinicalKey, from 2005 to 2015. Throughout the literary review, it was found that adherence assessment can be subjective or objective and that adherence has been evaluated with different types of instruments because of the great difficulty posed by the measurement of this variable. Multiple interfaces influence adherence to treatment, e.g., patient, disease, therapy, socioeconomic and cultural aspects, health system and relationship with healthcare professionals. Because the dimension of the theme of adherence is complex, there is no method or instrument that fits all the objectives and studies. However, methodological studies are needed to develop and validate instruments to assess adherence to nutritional orientations.

Keywords: Patient Compliance. Nutrition Therapy. Orientation. Evaluation. Guideline Adherence.

\section{Introdução}

As ações de educação nutricional têm lugar de destaque no tratamento de diversas doenças, como, por exemplo, das doenças cardiovasculares e pulmonares; e a adesão às orientações é imprescindível para que se obtenham os resultados esperados da educação alimentar. ${ }^{1}$

A adesão às orientações propostas influencia o sucesso do tratamento da doença e é um processo multifatorial que se estabelece mediante parceria entre o profissional da saúde e o paciente, englobando diversos aspectos, que vão desde a frequência dos atendimentos até o desenvolvimento da consciência para o autocuidado e manutenção da busca de saúde, formando atitudes, hábitos e práticas alimentares saudáveis., ${ }^{1,2}$

O tratamento de uma doença crônica, por exemplo, exige maior comprometimento do paciente e continuidade, o que justifica o fato de os pacientes crônicos terem menor adesão ao tratamento, que, muitas vezes, apresenta esquemas terapêuticos complexos. Estima-se que cerca de $50 \%$ ou mais 
desses pacientes não seguem o tratamento adequadamente. ${ }^{2-5} \mathrm{O}$ fenômeno da adesão e não adesão traz grande impacto na morbimortalidade de indivíduos que são acometidos por doenças crônicas. ${ }^{6}$

A baixa adesão ao tratamento é um dos problemas mais importantes enfrentados pelos profissionais de saúde. ${ }^{7,8}$ A não adesão pode ocasionar desnecessário ajuste na terapêutica devido à falta de respostas positivas, frustração dos profissionais de saúde, já que os objetivos traçados não são alcançados, e, sobretudo, aumento de custos no cuidado à saúde com elevação das taxas de hospitalização e tratamento de complicações. ${ }^{3,9}$

O impacto das doenças sobre as pessoas não pode ser descrito apenas por medidas objetivas de saúde, sendo assim, a criação e adaptação de instrumentos que possam captar de forma objetiva atributos subjetivos de diferentes temas relacionados ao processo saúde-doença-cuidado vêm sendo impulsionadas. Esses instrumentos podem proporcionar aproximação com a realidade, de modo a contribuir para intervenções mais efetivas, resultando no aumento da adesão. ${ }^{6}$

Percebe-se, assim, a relevância dos instrumentos de avaliação da adesão para a saúde pública e a necessidade de se preencher a lacuna em relação à adesão ao tratamento não farmacológico, já que a maioria dos métodos utilizados para avaliar a adesão é focada somente no tratamento farmacológico e já está bem estabelecido na literatura que há grande dificuldade de adesão ao tratamento não medicamentoso. ${ }^{3,10}$

O tratamento de diabetes, por exemplo, deveria iniciar com terapias não medicamentosas, como intervenções no estilo de vida, já que são mais efetivas para o controle da doença. ${ }^{11}$ Alimentação inadequada, fumo e falta de exercício físico são fatores de risco para doença arterial coronariana (DAC) e uma melhor adesão às mudanças de estilo de vida, após uma síndrome coronariana aguda, está associada a um menor risco de eventos cardiovasculares recorrentes. ${ }^{12}$ Hábitos alimentares saudáveis e prática regular de atividade física são ferramentas extremamente importantes na prevenção e tratamento de diversas doenças. ${ }^{13-16}$

Assim, reconhecendo a complexidade dessa questão, este estudo tem como objetivo identificar como é avaliada a adesão às orientações nutricionais e seus determinantes, além de sumarizar formas para melhorar a adesão.

\section{Método}

Trata-se de um estudo de revisão narrativa de literatura, norteado pela seguinte pergunta de pesquisa: como é avaliada a adesão às orientações nutricionais? Foi realizada uma busca de artigos nas bases de dados eletrônicas Scientific Electronic Library Online (SciELO), Literatura LatinoAmericana e do Caribe em Ciências da Saúde (LILACS), Public Medical (PubMed) e ClinicalKey. O período delimitado para a pesquisa foi de jan./2005 a jun./2015. Os seguintes descritores 
foram utilizados: "patient compliance", "nutrition therapy", "orientation”, "evaluation", "guideline adherence", e suas versões em português e espanhol, de acordo com a terminologia em saúde DeCS (Descritores em Ciências da Saúde), da Biblioteca Virtual em Saúde, e MeSH (Medical Subject Headings). As seguintes combinações de termos foram feitas: "patient compliance" and "nutrition therapy"; "guideline adherence" and "nutrition therapy"; "guideline adherence" and "evaluation"; "orientation" and "nutrition therapy"; "orientation" and "patient compliance".

Os critérios de inclusão adotados foram: artigos disponíveis na íntegra em língua portuguesa, espanhola ou inglesa, publicados no período delimitado, que abordavam a temática adesão e discutiam sobre o tema estabelecendo relação com o tratamento nutricional. Foram excluídos os artigos que citavam a palavra adesão, mas não discutiam sobre o tema, os artigos que abordavam a temática adesão, mas não evidenciaram de nenhuma forma como foi mensurada ou determinada a adesão, e os artigos que abordavam o tratamento farmacológico sem citar o tratamento não farmacológico. Dessa forma, foram selecionados 56 artigos de periódicos.

\section{Resultados e Discussão}

A adesão ao tratamento não tem uma definição estabelecida e universalmente aceita, com base nos tipos de dados, instrumentos e métodos de medição. ${ }^{5}$ Em termos de definição, avaliação e sobre os tipos de intervenções necessárias para aumentá-la, os estudos têm encontrado um grande desafio. ${ }^{17}$ Há uma grande dificuldade e variabilidade na definição de critérios que revelem adequadamente a adesão, o que torna complexo identificar a falta de adesão, assim como quantificá-la. , $, 6,9,10,18,19$

Diante dessa realidade, a adesão pode ser definida como uma situação em que o comportamento do paciente corresponde com as orientações dadas pelos profissionais de saúde; ou ainda, como uma participação ativa e de colaboração voluntária do paciente em comportamento aceito por mútuo acordo, a fim de produzir um resultado terapêutico ou preventivo desejado., ${ }^{6,17}$

Apesar da disponibilidade de tratamento efetivo para doenças crônicas como hipertensão e diabetes, o controle é difícil e encontra-se longe do considerado ideal pela falta de adesão às orientações propostas, o que influencia a inadequada evolução das doenças crônicas. ${ }^{4,10}$ Melhorar a adesão pode repercutir mais na saúde da população que qualquer outra melhora nos tratamentos médicos específicos, ${ }^{4}$ sendo o fator mais importante para o controle efetivo de diversas doenças. ${ }^{18}$

Os pacientes portadores de doenças crônicas nem sempre têm o discernimento imediato dos ganhos que os comportamentos de autocuidado podem proporcionar, o que pode contribuir para a não adesão ao tratamento. No caso de hipertensão arterial, o paciente, muitas vezes assintomático, não aceita que tem a doença, especialmente quando esse diagnóstico implica 
mudança de hábitos considerados prazerosos, construídos ao longo do tempo, ou ainda obrigação de usar medicamentos de forma permanente. ${ }^{20,21}$ Para aumentar a probabilidade de boa adesão do paciente ao tratamento, é importante que ele aprenda a estabelecer a relação entre o seguimento das orientações propostas e as consequências boas obtidas pelo seguimento destas. ${ }^{7}$ É imprescindível também uma transformação na maneira de pensar dos indivíduos e dos profissionais envolvidos nesse processo, ${ }^{11}$ pois perspectivas divergentes sobre a doença e tratamento entre pacientes e profissionais de saúde não facilitam a adesão. ${ }^{5}$ É imprescindível sensibilizar o paciente para adoção de novos hábitos e certificar-se de que ele entenda a importância das exigências impostas pelo tratamento, além do desenvolvimento de um bom relacionamento profissional-paciente.,11 A mudança de comportamento deve ser permanente e disso depende seu convívio amigável com a doença,${ }^{20}$ que não deve ser vista como um problema, mas como um desafio a ser enfrentado. ${ }^{14}$

O treino em correspondência é uma das técnicas utilizadas na área da saúde, em especial pelo psicólogo que segue o modelo analítico-comportamental, que auxilia o paciente a observar o próprio comportamento (auto-observação), com o objetivo de avaliar se as suas ações voltadas ao tratamento coincidem ou não com o que foi orientado pelos profissionais de saúde e verificar quais as variáveis que interferem na adesão, através de registros sistemáticos do próprio comportamento. Outra técnica é o relato verbal do paciente acerca de suas ações de autocuidado, em que é descrito oralmente as condutas-alvo. Embora possa haver omissão de condutas de não adesão, a literatura indica que dados confiáveis podem ser obtidos quando são relatadas condutas específicas em intervalos recentes de tempo, como os correspondentes às 24 horas anteriores ao procedimento de coleta de dados. ${ }^{7}$ Destarte, é importante destacar, para avaliação de consumo alimentar, a utilização de inquéritos alimentares como, por exemplo, o Recordatório Alimentar de 24 horas (R24h), que, apesar de suas limitações, como a subestimação do consumo de alimentos, é um instrumento de fácil uso, baixo custo e que pode ser utilizado em analfabetos. ${ }^{22,23}$ Através da coleta da informação de hábitos alimentares, pode-se descobrir quais mudanças devem ser feitas. ${ }^{24}$ Todavia, mensurar a adesão à dieta também é complexo devido à dificuldade de dimensionar as reais alterações na alimentação. $^{15}$

A adesão tem sido avaliada com diferentes tipos de ferramentas devido à grande dificuldade encontrada na maneira de mensurar essa variável, aliás, a avaliação da adesão ao tratamento não farmacológico não é avaliada como tal, mas geralmente integrada em comportamentos de autogestão ou atividades de autocuidado. ${ }^{5,25}$

A avaliação da adesão pode ser subjetiva ou objetiva. A subjetiva inclui a obtenção da informação do nível de adesão por meio de relato dos pacientes, familiares ou profissionais de saúde, através de questionários, diários ou entrevistas; registros e entrevistas são amplamente utilizados porque apresentam a avaliação do próprio paciente a respeito de sua adesão. ${ }^{17,25}$ Já as medidas objetivas contam com a precisão de dados objetivos, como medidas antropométricas e exames laboratoriais. ${ }^{25}$ 
As medidas subjetivas podem superestimar a adesão, já que as informações autorreferidas podem gerar erros decorrentes de memória ou outras distorções. ${ }^{15,25}$ No entanto, as técnicas subjetivas de autorrelato são de fácil emprego e podem permitir a identificação de um grupo de pacientes com comportamento de não adesão, ainda que com menor sensibilidade, o que auxilia na identificação de razões para essa atitude e no desenvolvimento de estratégias para melhorar a adesão. ${ }^{25}$ Especialmente no âmbito nutricional, o que tem sido feito é considerar a frequência relatada de comportamento alimentar, já que os métodos de autoavaliação são muito mais úteis que os métodos mais objetivos. ${ }^{17}$ Outra dificuldade da avaliação da adesão autorrelatada é o fato de não haver questionários validados para essa finalidade, por isso, o que muitos estudos fazem é utilizar vários questionários ou adaptar um questionário de outro trabalho, ou ainda desenvolver seu próprio questionário, para auxiliar na determinação do grau de adesão. ${ }^{25}$

Para Debussche, ${ }^{5}$ avaliar a adesão através de parâmetros bioquímicos é complexo, pois mudanças moderadas na alimentação ou prática de atividades físicas podem ocorrer sem nenhum efeito óbvio ser observado nos resultados biomédicos; portanto, a eficácia das intervenções educativas sobre a adesão e a relação entre a adesão e os critérios biomédicos ainda precisam ser esclarecidas.

Dentre os estudos analisados neste trabalho, os dados do quadro 1 resumem os principais métodos e instrumentos utilizados na avaliação da adesão constatados na busca bibliográfica.

Quadro 1. Tipo de estudo, instrumento utilizado, população de estudo, local/ano onde foram realizados, vantagens e/ou desvantagens do instrumento utilizado.

\begin{tabular}{|c|c|c|c|c|}
\hline Estudo & $\begin{array}{l}\text { Instrumento } \\
\text { adotado }\end{array}$ & $\begin{array}{l}\text { População de } \\
\text { estudo }\end{array}$ & Local/ano & $\begin{array}{l}\text { Vantagens e/ou } \\
\text { desvantagens do } \\
\text { instrumento }\end{array}$ \\
\hline $\begin{array}{l}\text { Bueno et al. } \\
\text { Retrospectivo, } \\
\text { longitudinal, } \\
\text { descritivo e } \\
\text { quantitativo }\end{array}$ & $\begin{array}{l}\text { Permanência do } \\
\text { indivíduo até o } \\
\text { último encontro, } \\
\text { além de medidas } \\
\text { antropométricas } \\
\text { e registros } \\
\text { alimentares }\end{array}$ & $\begin{array}{l}\text { Adultos com } \\
\text { excesso de peso } \\
(\mathrm{n}=116)\end{array}$ & $\begin{array}{l}\text { Comunidade } \\
\text { universitária } \\
\text { pública de } \\
\text { Ribeirão Preto } \\
\text { (SP)-Brasil/2011 }\end{array}$ & $\begin{array}{l}\text { Parâmetros } \\
\text { antropométricos: simples, } \\
\text { de baixo custo, porém } \\
\text { podem resultar em erro } \\
\text { sistemático na estimativa } \\
\text { da composição corporal, } \\
\text { além da necessidade de } \\
\text { avaliadores treinados. } \\
\text { Registro alimentar: não } \\
\text { depende da memória, } \\
\text { porém o consumo pode ser } \\
\text { alterado e exige alto nível } \\
\text { de colaboração }\end{array}$ \\
\hline
\end{tabular}

continua 
Continuação do Quadro 1

\begin{tabular}{|c|c|c|c|c|}
\hline Estudo & $\begin{array}{l}\text { Instrumento } \\
\text { adotado }\end{array}$ & $\begin{array}{c}\text { População de } \\
\text { estudo }\end{array}$ & Local/ano & $\begin{array}{l}\text { Vantagens e/ou } \\
\text { desvantagens do } \\
\text { instrumento }\end{array}$ \\
\hline $\begin{array}{l}\text { Figueiredo e } \\
\text { Asakura } \\
\text { Descritivo } \\
\text { de corte } \\
\text { transversal }\end{array}$ & $\begin{array}{l}\text { Questionário } \\
\text { com perguntas } \\
\text { abertas e } \\
\text { fechadas }\end{array}$ & $\begin{array}{l}\text { Pacientes } \\
\text { hipertensos } \\
(n=54)\end{array}$ & $\begin{array}{l}\text { Centro de Saúde } \\
\text { de São Paulo- } \\
\text { Brasil/2010 }\end{array}$ & $\begin{array}{l}\text { Alguns itens podem } \\
\text { representar conceitos } \\
\text { amplos }\end{array}$ \\
\hline $\begin{array}{l}\text { Girotto et al. } \\
\text { Transversal, } \\
\text { exploratório }\end{array}$ & $\begin{array}{l}\text { Autorrelato } \\
\text { por meio de } \\
\text { entrevista }\end{array}$ & $\begin{array}{l}\text { Indivíduos } \\
\text { hipertensos } \\
(\mathrm{n}=380)\end{array}$ & $\begin{array}{l}\text { Área de } \\
\text { abrangência de } \\
\text { uma Unidade de } \\
\text { Saúde da } \\
\text { Família de } \\
\text { Londrina (PR)- } \\
\text { Brasil/2013 }\end{array}$ & $\begin{array}{l}\text { Fácil emprego e pode } \\
\text { permitir a identificação } \\
\text { de um grupo de pacientes } \\
\text { com comportamento } \\
\text { de não adesão. No } \\
\text { entanto, pode gerar erros } \\
\text { decorrentes de memória } \\
\text { ou outras distorções }\end{array}$ \\
\hline $\begin{array}{l}\text { Silva et al. } \\
\text { Longitudinal } \\
\text { de intervenção }\end{array}$ & $\begin{array}{l}\text { Parâmetros } \\
\text { dietéticos, } \\
\text { antropométricos, } \\
\text { bioquímicos e } \\
\text { clínicos }\end{array}$ & $\begin{array}{l}\text { Mulheres } \\
\text { hipertensas } \\
(\mathrm{n}=27)\end{array}$ & $\begin{array}{l}\text { Unidade } \\
\text { de Atenção } \\
\text { Primária à } \\
\text { Saúde de Ponte } \\
\text { Firme (MG)- } \\
\text { Brasil/2014 }\end{array}$ & $\begin{array}{l}\text { Precisão de dados } \\
\text { objetivos. Entretanto, } \\
\text { mudanças moderadas na } \\
\text { alimentação ou prática de } \\
\text { atividades físicas podem } \\
\text { ocorrer sem nenhum efeito } \\
\text { óbvio ser observado nos } \\
\text { resultados biomédicos, ou } \\
\text { vice-versa }\end{array}$ \\
\hline $\begin{array}{l}\text { Campuzano } \\
\text { et al. } \\
\text { Pré-teste/pós- } \\
\text { teste }\end{array}$ & $\begin{array}{l}\text { Registros } \\
\text { alimentares de } \\
\text { três semanas }\end{array}$ & $\begin{array}{l}\text { Pacientes } \\
\text { diabéticos } \\
(\mathrm{n}=90)\end{array}$ & $\begin{array}{l}\text { Centros de } \\
\text { saúde da cidade } \\
\text { do México/2013 }\end{array}$ & $\begin{array}{l}\text { Identifica tipos de } \\
\text { alimentos e preparações } \\
\text { consumidos e } \\
\text { horários das refeições. No } \\
\text { entanto, requer tempo e o } \\
\text { indivíduo deve conhecer } \\
\text { medidas caseiras }\end{array}$ \\
\hline $\begin{array}{l}\text { Guimarães et } \\
\text { al. } \\
\text { Intervenção } \\
\text { controlada e } \\
\text { aberta }\end{array}$ & $\begin{array}{l}\text { Repercussões } \\
\text { dietéticas } \\
\text { antes e após a } \\
\text { intervenção e } \\
\text { assiduidade às } \\
\text { reuniões }\end{array}$ & $\begin{array}{l}\text { Portadores de } \\
\text { dois ou mais } \\
\text { fatores de risco } \\
\text { cardiovasculares } \\
\text { associados } \\
(n=80)\end{array}$ & $\begin{array}{l}\text { Ambulatório de } \\
\text { nutrição de um } \\
\text { hospital público } \\
\text { de Brasília- } \\
\text { Brasil/2010 }\end{array}$ & $\begin{array}{l}\text { Maior número de } \\
\text { consultas está relacionado } \\
\text { a uma adesão mais alta }\end{array}$ \\
\hline
\end{tabular}


Continuação do Quadro 1

\begin{tabular}{|c|c|c|c|c|}
\hline Estudo & $\begin{array}{l}\text { Instrumento } \\
\text { adotado }\end{array}$ & $\begin{array}{c}\text { População de } \\
\text { estudo }\end{array}$ & Local/ano & $\begin{array}{l}\text { Vantagens e/ou } \\
\text { desvantagens do } \\
\text { instrumento }\end{array}$ \\
\hline $\begin{array}{l}\text { Dalcin et al. } \\
\text { Transversal, } \\
\text { prospectivo }\end{array}$ & $\begin{array}{l}\text { Autorrelato } \\
\text { por meio de } \\
\text { questionário } \\
\text { adaptado }\end{array}$ & $\begin{array}{l}\text { Pacientes com } \\
\text { fibrose cística } \\
(\mathrm{n}=38)\end{array}$ & $\begin{array}{l}\text { Hospital público } \\
\text { de Porto Alegre } \\
\text { (RS)-Brasil/2005 } \\
\text { a } 2007\end{array}$ & $\begin{array}{l}\text { Não há questionários } \\
\text { validados para essa } \\
\text { finalidade }\end{array}$ \\
\hline $\begin{array}{l}\text { Mussi et al. } \\
\text { Transversal }\end{array}$ & $\begin{array}{l}\text { Questionário } \\
\text { desenvolvido } \\
\text { e validado no } \\
\text { Brasil para uso } \\
\text { em pacientes } \\
\text { com IC }\end{array}$ & $\begin{array}{l}\text { Pacientes com } \\
\text { insuficiência } \\
\text { cardíaca } \\
(\mathrm{n}=340)\end{array}$ & $\begin{array}{l}\text { Duas instituições } \\
\text { de referência } \\
\text { nas cidades de } \\
\text { Porto Alegre } \\
\text { (RS) e Niterói } \\
\text { (RJ)-Brasil/2015 }\end{array}$ & $\begin{array}{l}\text { Fácil emprego. Pode gerar } \\
\text { erros decorrentes de } \\
\text { distorções }\end{array}$ \\
\hline $\begin{array}{l}\text { Nascimento } \\
\text { et al. } \\
\text { Transversal }\end{array}$ & $\begin{array}{l}\text { Autorrelato do } \\
\text { seguimento ou } \\
\text { não de um plano } \\
\text { dietético }\end{array}$ & $\begin{array}{l}\text { Pacientes } \\
\text { diabéticos } \\
(\mathrm{n}=34)\end{array}$ & $\begin{array}{l}\text { Hospital público } \\
\text { de Curitiba } \\
\text { (PR)-Brasil/2014 }\end{array}$ & $\begin{array}{l}\text { Fácil aplicação, porém } \\
\text { depende de memória ou } \\
\text { pode gerar distorções }\end{array}$ \\
\hline $\begin{array}{l}\text { García- } \\
\text { Meseguer et al. } \\
\text { Transversal }\end{array}$ & $\begin{array}{l}\text { Dois R24h não } \\
\text { consecutivos, } \\
\text { HEI e Escore } \\
\text { da Dieta } \\
\text { Mediterrânea }\end{array}$ & $\begin{array}{l}\text { Estudantes } \\
(\mathrm{n}=284)\end{array}$ & $\begin{array}{l}\text { Universidade } \\
\text { espanhola, } \\
\text { Albacete- } \\
\text { Espanha/2014 }\end{array}$ & $\begin{array}{l}\text { R24h: instrumento de fácil } \\
\text { utilização, baixo custo e } \\
\text { que pode ser utilizado em } \\
\text { analfabetos. Apresenta } \\
\text { limitações. como a } \\
\text { subestimação do consumo } \\
\text { de alimentos. Escores de } \\
\text { dieta: avaliam a qualidade } \\
\text { da dieta individual e } \\
\text { populacional em termos } \\
\text { de conformidade com } \\
\text { a orientação dietética. } \\
\text { Requerem entrevistadores } \\
\text { treinados }\end{array}$ \\
\hline
\end{tabular}

continua 
Continuação do Quadro 1

\begin{tabular}{|c|c|c|c|c|}
\hline Estudo & $\begin{array}{l}\text { Instrumento } \\
\text { adotado }\end{array}$ & $\begin{array}{c}\text { População de } \\
\text { estudo }\end{array}$ & Local/ano & $\begin{array}{l}\text { Vantagens e/ou } \\
\text { desvantagens do } \\
\text { instrumento }\end{array}$ \\
\hline $\begin{array}{l}\text { Torrado et al. } \\
\text { Longitudinal }\end{array}$ & $\begin{array}{l}\text { Questionário } \\
\text { MEDAS }\end{array}$ & $\begin{array}{l}\text { Pessoas com } \\
\text { excesso de peso } \\
(n=99)\end{array}$ & $\begin{array}{l}\text { Unidade de } \\
\text { saúde que } \\
\text { enfatiza a } \\
\text { prestação } \\
\text { de serviços } \\
\text { farmacêuticos } \\
\text { em Madrid- } \\
\text { Espanha/2014 }\end{array}$ & $\begin{array}{l}\text { Adesão ao padrão } \\
\text { alimentar Mediterrâneo }\end{array}$ \\
\hline $\begin{array}{l}\text { Gopinath et al. } \\
\text { Coorte }\end{array}$ & $\begin{array}{l}\text { QFA no início do } \\
\text { estudo e escores } \\
\text { totais da dieta }\end{array}$ & $\begin{array}{l}1.305 \text { e } 895 \\
\text { participantes } \\
\text { com dados } \\
\text { completos } \\
\text { depois de cinco } \\
\text { e dez anos, } \\
\text { respectivamente }\end{array}$ & $\begin{array}{l}\text { Sydney- } \\
\text { Austrália/2014 }\end{array}$ & $\begin{array}{l}\text { QFA: método de baixo } \\
\text { custo e relativamente } \\
\text { simples. Depende da } \\
\text { memória. Não estima o } \\
\text { consumo absoluto. Escores } \\
\text { de dieta: também estudam } \\
\text { os benefícios para a saúde } \\
\text { de um determinado } \\
\text { padrão alimentar. } \\
\text { Requerem entrevistadores } \\
\text { treinados }\end{array}$ \\
\hline $\begin{array}{l}\text { Fung et al. } \\
\text { Coorte } \\
\text { prospectivo }\end{array}$ & $\begin{array}{l}\text { QFA no início } \\
\text { do estudo e } \\
\text { escore com base } \\
\text { nos alimentos } \\
\text { enfatizados ou } \\
\text { minimizados } \\
\text { pela dieta DASH }\end{array}$ & $\begin{array}{l}\text { Enfermeiras } \\
(\mathrm{n}=88.517)\end{array}$ & $\begin{array}{l}11 \text { estados dos } \\
\text { EUA/2008 }\end{array}$ & - \\
\hline
\end{tabular}

R24h: Recordatório Alimentar de 24 horas; HEI: Healthy Eating Index; MEDAS: Mediterranean Diet Adherence Screener; QFA: questionário de frequência alimentar; DASH: Dietary Approaches to Stop Hypertension 
Vários estudos têm discutido abordagens educacionais para melhorar a adesão ao tratamento de pacientes com insuficiência cardíaca (IC). ${ }^{26-30}$ A terapia nutricional da IC está diretamente relacionada a esquemas terapêuticos complexos que incluem restrições dietéticas de sal e líquidos. Além disso, a alta prevalência de DAC como uma causa de IC faz necessária a restrição da ingestão de gordura saturada, colesterol e carboidratos simples na dieta desses pacientes. ${ }^{26,27}$ Essa complexidade do tratamento dificulta ainda mais o seguimento do plano terapêutico pelo paciente, já que é estabelecida a relação de quanto maior a complexidade, menor será a adesão terapêutica. ${ }^{17}$

O tratamento não farmacológico em pacientes com IC é importante para manter a estabilidade, evitar readmissão hospitalar e diminuir mortalidade. ${ }^{27,29}$ As taxas de não adesão a esse tratamento também são elevadas (entre $50 \%$ e $80 \%$ ), e entre as maiores limitações para a adesão, destaca-se o desconhecimento e a falta de associação entre o que os portadores de IC sabem o que precisa ser feito e o resultado de determinado comportamento, ou seja, eles compreendem que uma dieta com pouco sal é importante, mas nem sempre relacionam o excesso de sal na dieta à retenção hídrica e ao aumento de peso, que podem propiciar descompensação clínica e consequente readmissão hospitalar. ${ }^{26,30}$

O esquecimento é uma importante barreira para a adesão e há associação significativa entre IC e disfunção cognitiva, sendo esta prevalente entre os idosos. ${ }^{26,30}$ De maneira geral, a idade avançada favorece uma baixa adesão ao tratamento, seja devido à presença de várias doenças crônicas ou à diminuição da capacidade cognitiva e funcional. ${ }^{22}$

Um ensaio clínico randomizado, ${ }^{28}$ denominado Home based intErvention LEd by Nurse in Brazil (HELEN-I), verificou o efeito de uma intervenção educativa de enfermagem, resultante da combinação entre visita domiciliar e contato telefônico, em pacientes com internação recente por IC descompensada, no conhecimento da doença, nas habilidades de autocuidado e na adesão ao tratamento, comparado ao acompanhamento convencional de pacientes, no período de seis meses. Foram randomizados 200 pacientes, com melhora significativa no conhecimento e autocuidado para o grupo intervenção. Para aferir o conhecimento sobre a IC, foi aplicado um questionário composto de 14 questões e para medir as habilidades para o autocuidado, utilizada a European Heart Failure Self Care Behaviour Scale (EHFScBS), composta por 12 questões; ambos instrumentos validados para uso no Brasil. ${ }^{31,32}$ A adesão ao tratamento foi aferida no final entre os grupos através de um questionário também validado composto por dez questões. As perguntas incluem uma sobre o uso de medicação, uma sobre o controle de peso, três sobre os hábitos alimentares, três sobre ingestão de líquidos, uma sobre o consumo de bebidas alcoólicas e uma sobre o comparecimento às consultas agendadas. $\mathrm{O}$ escore de acertos varia de 0 a 26 pontos; quanto maior a pontuação, mais alta a adesão. Foi considerada adesão adequada quando o paciente atingia um escore de 18 pontos, o que correspondia a 70\%. Nesse estudo, a adesão foi significativamente maior no grupo intervenção, que recebeu visitas domiciliares e contato telefônico para reforço das orientações. 
Ainda não há consenso a respeito de um padrão que constitui uma taxa de adesão adequada para o tratamento de doenças crônicas; todavia, alguns estudos, com enfoque para o tratamento farmacológico, sugerem que taxas em torno de $80 \%$ são aceitáveis. ${ }^{18}$

O mesmo questionário citado acima foi utilizado para avaliar a adesão ao tratamento em um estudo transversal ${ }^{29}$ realizado em duas clínicas especializadas no atendimento ambulatorial para pacientes com IC crônica, localizadas na região Sul (Porto Alegre) e região Sudeste (Niterói) do Brasil. Esse estudo analisou a adesão ao tratamento de IC em 340 pacientes acompanhados pela equipe de enfermagem e sua associação com características dos mesmos, como número de consultas anteriores, estrutura familiar e comorbidades. A média do escore de adesão foi de 16 ( \pm 4$)$ pontos e apenas $124(36,5 \%)$ deles apresentaram uma taxa de adesão $\geq 70 \%$. Ademais, demonstrou-se que os pacientes que viviam com suas famílias tiveram escores de adesão mais elevados e que três ou mais consultas de enfermagem anteriores estavam significativamente associadas com uma adesão mais alta. A presença de hipertensão como uma comorbidade foi relacionada com baixa adesão ao tratamento.

Outro instrumento que pode ser utilizado para auxiliar no desenvolvimento de estratégias de educação e tratamento em paciente com IC é o Dietary Sodium Restriction Questionnaire (DSRQ), que tem como objetivo identificar fatores que afetam a adesão à restrição dietética de sódio para esses pacientes. Uma adaptação transcultural para o Brasil desse questionário, com posterior validação, foi realizada por d'Almeida et al. ${ }^{33,34}$

Compreender informações básicas de saúde é fundamental para realização de mudanças de estilo de vida e, segundo Alves et al., ${ }^{27}$ seria o passo inicial para melhorar a adesão do paciente ao tratamento dietético, que poderia ser focado no aumento do conhecimento sobre o que é considerada uma alimentação saudável. O conhecimento sobre o tratamento da doença também tem sido avaliado como preditor da adesão dietética, ${ }^{35} \mathrm{e}$, apesar de ser algo racional e a adesão um processo complexo que engloba diversos fatores biossociais e emocionais, com o que concorda Jesus et al., ${ }^{9}$ deve ser considerado no contexto da adesão ao tratamento. Cabe destacar que levar o conhecimento às pessoas é importante, mas a relação entre o saber o que é preciso e o fazer é frágil, já que o conhecimento não instiga a mudança, porém, quando os indivíduos estão estimulados, desejam mudar, pode funcionar como um instrumento de mudança, constituindo o primeiro degrau do comportamento saudável. ${ }^{1,21}$

Um estudo metodológico ${ }^{36}$ realizou adaptação transcultural e validação para uso no Brasil de um questionário de conhecimento dos fatores de risco cardiovascular (Q-FARCS), mudança do estilo de vida e adesão, em uma amostra de pacientes com DAC. O questionário é dividido em três escores: conhecimento geral dos fatores de risco, conhecimento específico e mudanças no estilo de vida. Uma vez que o conhecimento pode se correlacionar com o comportamento do paciente em relação a alguns fatores de risco, determinar seu grau pode ser útil na tomada de decisão quanto 
à intensidade das intervenções e na orientação individualizada do paciente. Logo, instrumentos que meçam o conhecimento como um meio de avaliar a adesão também podem ser úteis na busca para aumento da adesão às orientações propostas.

A adesão ao tratamento por hipertensos por si só é considerada baixa, e quando associada com IC diminui ainda mais, já que o número de medicamentos aumenta, assim como elevam os custos do tratamento e as restrições de estilo de vida. ${ }^{29}$ No que se refere à hipertensão arterial, existem vários instrumentos para mensurar a adesão ao tratamento, como o Cuestionário de valoración del comportamiento de adherencia al tratamiento farmacológico y no farmacológico, o Questionário MBG (Martín-Bayarre-Grau), a Hill-Bone compliance to high blood pressure therapy scale, entre outros. Esses questionários medem diferentes construtos relacionados à adesão, porém muitos estão validados apenas em espanhol e em língua inglesa e, sobretudo, a maioria enfoca o tratamento medicamentoso. ${ }^{6}$

Em contraste com o acima exposto, destaca-se o "questionário de adesão ao tratamento da hipertensão arterial sistêmica" (QATHAS), elaborado e validado no Brasil por Rodrigues et al., ${ }^{3}$ que buscou avaliar a adesão ao tratamento farmacológico e não farmacológico. O QATHAS possui 12 questões, das quais cinco são sobre o tratamento farmacológico (uso de medicação, dose da medicação, horário da medicação, sintoma, rotina do tratamento medicamentoso), quatro sobre os hábitos alimentares (uso do sal, uso da gordura, consumo de carnes brancas, consumo de doces e bebidas com açúcar), uma sobre exercício físico, uma sobre seguir o tratamento não medicamentoso e uma sobre o comparecimento às consultas agendadas. Ao responder o QATHAS, diferentemente dos outros questionários, o resultado não é obtido ao somar pontos ou escores, mas por fórmula matemática, que determina o nível de adesão em que cada respondente está situado, que varia de 60 a 110, isto é, os hipertensos com menor adesão atingem 60, enquanto os mais aderentes situam-se no nível 110.

Figueiredo \& Asakura ${ }^{10}$ investigaram, através de um estudo descritivo de corte transversal, as principais dificuldades de pacientes hipertensos em seguimento ambulatorial para aderir ao tratamento proposto pela equipe de saúde. Foram entrevistados 54 indivíduos com um roteiro preestabelecido, contendo perguntas abertas e fechadas sobre características sociodemográficas, hábitos de vida e o tratamento para hipertensão e o que eles sabiam sobre a doença. A principal dificuldade relatada no tratamento foi a adoção de uma dieta hipossódica, por não gostarem do sabor ou porque recebiam sua alimentação com sal preparada por outra pessoa, seguida da realização regular de atividade física por razões como dor, falta de companhia e de tempo. Em relação ao tratamento medicamentoso, a principal dificuldade foi lembrar o horário para tomar a medicação. Observaram ainda associação entre o conhecimento da doença e o seguimento das orientações recebidas, mas não entre o conhecimento ou o seguimento das orientações com o controle de pressão arterial, demonstrando que, assim como no estudo de Ribeiro et al., ${ }^{37} \mathrm{o}$ conhecimento sobre a doença não necessariamente implica uma mudança de comportamento 
e, apesar de fundamental para a adesão do paciente, por si só não é o suficiente para promover mudanças necessárias para melhorar a adesão à dieta, ${ }^{17,38}$ o que corrobora os achados na literatura.

O diabetes está entre as doenças com menores taxas de adesão ao tratamento por exigir diversos cuidados. ${ }^{39}$ Programas educacionais para indivíduos com essa doença têm sido promovidos visando à otimização do tratamento, onde os participantes são instruídos sobre ela a fim de obtenção de autonomia para o seu controle. Porém, a literatura mostra que apenas a informação transmitida no tratamento não assegura a adesão do paciente. ${ }^{7}$ Assim, de acordo com Ferreira \& Fernandes, ${ }^{7}$ os profissionais de saúde têm procurado aliar o conhecimento das orientações ao seu seguimento.

Uma revisão sistemática ${ }^{35}$ de 23 artigos sobre adesão à dieta em jovens com diabetes tipo 1 listou diferentes estratégias utilizadas nos estudos para medir a adesão, incluindo R24h, Questionários de Frequência Alimentar (QFA), registros alimentares padronizados e medidas de adesão padronizadas. Essa revisão mostrou taxas de adesão a comportamentos alimentares que variam de $21 \%$ a $95 \%$, dependendo do comportamento específico estudado e do tipo de dieta prescrita, mais flexível ou não em relação ao controle de carboidratos.

Davison et al. ${ }^{13}$ averiguaram a relação da adesão à dieta, controle glicêmico e fatores de risco cardiovascular em 3.180 pacientes brasileiros com diabetes tipo 1. A adesão foi definida como o seguimento de pelo menos $80 \%$ do tempo à dieta prescrita, relatada pelos pacientes através de questões autorreferidas relacionadas com fatores nutricionais incluídas em um questionário aplicado durante uma visita clínica ambulatorial. Esse estudo mostrou que 54,2\% dos indivíduos foram aderentes à dieta, com melhor controle de glicemia, peso e perfil lipídico dos pacientes que relataram adesão quando comparados àqueles que não eram aderentes. Além disso, os aderentes reportaram menor dificuldade de seguimento da dieta. As dificuldades mais frequentes relatadas foram em relação à quantidade de alimentos e ao fracionamento da dieta prescrita, seguidas de evitar açúcar e doces, compreensão da lista de substituições de alimentos e comer hortaliças e frutas.

Nascimento et al. ${ }^{39}$ verificaram a adesão à terapia nutricional como forma de tratamento em 34 pacientes diabéticos, adultos e idosos, internados em um hospital público de Curitiba, por meio da aplicação de um questionário que incluía perguntas a respeito da terapia nutricional de forma subjetiva, direcionadas aos próprios , que abordavam ou não o seguimento de um planejamento dietético e quem havia realizado a prescrição. Do total, 58,8\% dos pacientes afirmaram realizar terapia nutricional como forma de tratamento, porém grande parte não possuía prescrição dietética de profissional habilitado, no caso, o nutricionista, e metade realizava dieta com base na mídia e na cultura popular; 41,2\% relataram não realizar nenhum tipo de controle alimentar. Dos pacientes que possuíam prescrição dietética de profissional de saúde, $50 \%$ disseram seguir todas as recomendações, e $50 \%$ responderam seguir parcialmente, e as dificuldades mais citadas foram a redução no consumo de doces e de massas. Ademais, dos pacientes que realizavam a terapia nutricional com orientação do profissional de saúde, $90 \%$ relataram sentir modificações positivas 
após terem iniciado a dieta e $100 \%$ afirmaram que indicariam a terapia para outros pacientes. Os autores observaram ainda que os pacientes não realizavam o tratamento de forma conjunta, sendo a terapia medicamentosa a mais citada, seguida da terapia dietética, a prática de atividades físicas era realizada apenas por uma pequena parcela, sendo justificada em alguns casos pela presença de outras doenças que dificultavam realização de exercícios.

Assunção \& Ursine ${ }^{40}$ investigaram a associação entre fatores educacionais, demográficos, socioeconômicos, de saúde, percepção da doença, suporte social e adesão ao tratamento não farmacológico em 164 portadores de diabetes mellitus assistidos pelo Programa Saúde da Família (PSF) em um bairro de Belo Horizonte. Foi desenvolvido um questionário semiestruturado para obter informações referentes às variáveis estudadas, e a adesão ao tratamento foi definida por resposta positiva em ambas as perguntas: "Faz algum tipo de atividade física regular?" e "Faz algum tipo de controle alimentar?". As autoras consideraram adesão parcial por resposta positiva em uma das perguntas (atividade física regular ou controle alimentar) e nenhuma adesão por resposta negativa em ambas as perguntas. Verificou-se associação significante entre a adesão ao tratamento não farmacológico e baixa renda, local de residência, conhecimento sobre complicações, motivação com o tratamento, fazer parte de grupo de diabéticos e orientações recebidas pelo enfermeiro e fisioterapeuta.

Dos instrumentos utilizados para avaliar a aderência às atividades de autocuidado no paciente diabético, destaca-se o Summary of Diabetes Self-Care Activities Questionnaire (SDSCA), que foi traduzido e adaptado culturalmente para o Brasil por Michels et al., ${ }^{41}$ denominando-se "Questionário de Atividades de Autocuidado com o Diabetes" (QAD). O QAD possui seis dimensões e 15 itens de avaliação do autocuidado com o diabetes: "alimentação geral" (com dois itens), "alimentação específica" (três itens), "atividade física" (dois itens), "monitorização da glicemia" (dois itens), "cuidado com os pés" (três itens) e "uso da medicação" (três itens, utilizados de acordo com o esquema medicamentoso). Alem disso, possui outros três itens para avaliação do tabagismo. Quando avaliados com o questionário, os pacientes relatam com que frequência eles realizaram as atividades ou os comportamentos nos sete dias anteriores. As respostas variam de 0 a 7 , com os escores indicando o nível de aderência às atividades de autocuidado. No instrumento, zero representa a situação menos desejável e sete, a mais desejável, exceto na dimensão alimentação específica, em que os valores são invertidos. Apesar da aplicação do questionário ser fácil, prática e rápida, como todo instrumento o QAD apresenta limitações, como itens que representam conceitos amplos que podem ser difíceis de avaliar ou interpretar, por exemplo, o item "seguir uma dieta saudável”. Entretanto, sua aplicação pode auxiliar na melhor adesão, pois recorda os pacientes sobre os cuidados essenciais em seu tratamento.

Ainda é difícil traçar o perfil do paciente não aderente. ${ }^{30}$ Entretanto, fatores pessoais dos indivíduos também incidem negativamente sobre a adesão, como falta de motivação para modificação de estilo de vida, falta de conhecimento acerca da doença, tanto dos cuidadores quanto 
dos próprios pacientes, baixa autoestima ou depressão, ausência de apoio familiar, problemas pessoais, elevado número de doenças associadas, ausência de sintomas, estresse, representações negativas em relação à doença e ao tratamento, bem como a passividade do paciente em relação aos profissionais de saúde e à escolha do regime terapêutico. 8,11,17,19,21,22,40

Entre os fatores facilitadores da adesão estão: tratamento flexível, maior tempo de diagnóstico, simplificação das prescrições e objetivos claros, com enfoque em mudanças graduais, apoio social e familiar, escolaridade do paciente, sentimento de bem-estar, maior número de consultas., 4,9,24,27

A família pode ter um papel ambivalente em relação às mudanças alimentares, pois ao mesmo tempo que pode dificultar na mudança de comportamento pela resistência às modificações, é uma parte ativa e integrante da mudança, incentivando e motivando os pacientes. ${ }^{5,30}$ Dessarte, as propostas de mudanças alimentares devem levar em consideração o ambiente familiar e social, uma vez que as práticas relativas à alimentação são determinadas e influenciadas nesse convívio e fatores inseridos nesse contexto relacionam-se à adesão. ${ }^{8,19}$

Com o que concordam Maffacciolli \& Lopes citado por Franzoni et al, ${ }^{42}$ resistir a mudanças é um processo que está naturalmente presente nas relações humanas. A motivação para a mudança é afetada por diversas condições externas ao paciente, sendo que o indivíduo depois de motivado percorre as etapas dos cinco estágios de mudança comportamental estabelecidos, descritos como pré-contemplação, contemplação, preparação, ação e manutenção. Entende-se, a partir deste modelo, denominado Modelo Transteórico, que o indivíduo está pronto para efetivar alguma mudança apenas quando atinge o quarto estágio, que seria o momento no qual a pessoa inicia, ativamente, a mudança de hábito. ${ }^{42,43}$ A investigação do comportamento alimentar através da identificação dos estágios motivacionais possibilita aumentar a efetividade das intervenções nutricionais, já que à medida que se conhecem melhor os determinantes do comportamento alimentar, aumentam as chances de sucesso da terapêutica. ${ }^{43}$

Frazoni et al. ${ }^{42}$ comprovaram que a motivação é essencial para possibilitar mudança de hábitos, por meio de seu estudo experimental, de natureza qualiquantitativa, que visou avaliar se a intervenção nutricional em grupo modifica hábitos alimentares. Entretanto, nota-se nesse estudo que a melhora na mudança dos hábitos alimentares durante a intervenção (encontros semanais em um período de um mês), identificada através do questionário "Como está a sua alimentação?", do Ministério da Saúde, não se consolidou após o término da intervenção em grupo, o que indica a necessidade da manutenção da educação em saúde.

Algo semelhante pode ser encontrado no estudo de Schultz et al., ${ }^{14}$ com mulheres com fatores de risco para síndrome metabólica, em que demonstraram que a intervenção educativa de curto prazo, com atendimentos nutricionais individualizados e oficinas educativas quinzenais, por quatro meses, foi eficaz para estimular a mudança qualitativa da alimentação. Entretanto, foi 
observada, em uma avaliação realizada após seis meses, uma tendência de abandono dos hábitos alimentares recomendados ao longo da intervenção, ressaltando a necessidade de intervenções periódicas de manutenção.

A adesão é um grande e complexo desafio para a saúde pública, que envolve um processo comportamental multidimensional influenciado por vários fatores, como o próprio indivíduo, o estado de saúde, a terapia, os aspectos socioeconômicos, o sistema de saúde e o relacionamento com os profissionais de saúde, englobando dimensões biológica, psicológica, socioeconômica e cultural. ${ }^{1,37,44} \mathrm{~A}$ adesão ao tratamento não farmacológico parece ser mais difícil. ${ }^{8,10} \mathrm{~A}$ adesão às orientações nutricionais é frequentemente mais baixa que a adesão ao tratamento farmacológico, o que parece melhorar quando os pacientes recebem orientação de uma equipe multidisciplinar.,27

A adesão a programas de intervenção destinados a promover mudanças de estilo de vida em pacientes obesos também é baixa. ${ }^{4}$ A maioria dos obesos tem hábitos inadequados que proporcionam o desenvolvimento dessa doença e, segundo Golay citado por Rodríguez et al., ${ }^{24} 90 \%$ das pessoas que tentam perder peso fracassam. Assim sendo, para Rodríguez et al., ${ }^{24}$ as mudanças devem ser feitas de forma gradual apenas em alimentos ou grupos de alimentos que estão sendo consumidos de maneira inadequada, o que poderia facilitar a adesão à dieta proposta. Ainda, Alves et al., ${ }^{27}$ em concordância com Rodríguez et al., ${ }^{24}$ afirmam que a orientação nutricional deve ser preferencialmente individualizada, com uma dieta específica ao perfil fisiológico e comportamental do paciente, tendo assim mais possibilidade de sucesso, levando em consideração a complexidade do tratamento de determinada doença. Ao que Lustosa et al. ${ }^{18}$ corroboram, referindo que tratar todos os pacientes com a mesma doença da mesma forma contribui para menor adesão ao tratamento.

Moreira et al. ${ }^{45}$ avaliariam, em estudo transversal, descritivo e quantitativo, a evolução antropométrica e nutricional, observando a redução de peso e circunferência abdominal e a mudança no padrão alimentar de 78 pacientes obesos, de ambos os sexos, em atendimento nutricional em grupo, acompanhados quinzenalmente durante seis meses. Entre os 78 pacientes encaminhados para o atendimento em grupo, 59 compareceram ao primeiro encontro e apenas 40 chegaram ao final do programa. Os autores sugerem que a taxa de abandono encontrada no estudo pode ter sido decorrente ao fato de que os participantes não se encontrassem em estágios favoráveis para mudança de comportamento e, por conseguinte, não estariam preparados ou motivados para a ação, isto é, para a mudança de hábitos e, consequentemente, para a redução de peso. Com base nessa perspectiva, destacam ainda a importância da abordagem psicológica para reafirmar os objetivos do tratamento para perda de peso e motivar os pacientes. Apesar da taxa de desistência, o programa proposto mostrou-se muito eficaz para a redução de peso e reeducação alimentar.

Vivas et al. ${ }^{4}$ identificaram variáveis preditoras de baixa adesão a um programa piloto de modificação de estilos de vida baseado em dieta equilibrada hipocalórica, promoção do exercício 
físico e apoio psicológico, dirigido a indivíduos com excesso de peso em atenção primária à saúde. Baixa adesão ao programa foi considerada quando a participação dos indivíduos era inferior a $80 \%$ dos encontros quinzenais. Ao final da intervenção, $55 \%$ dos participantes apresentaram baixa adesão, e as causas autorrelatadas de não adesão ou descontinuação do tratamento foram: férias, problemas de trabalho, falta de motivação e problemas familiares. As variáveis identificadas como preditoras de baixa adesão foram relacionadas a ter Índice de Massa Corporal (IMC) $\geq 35 \mathrm{~kg} / \mathrm{m}^{2}$, deixado de fumar em um período $\leq 4$ meses, realizar dieta hipercalórica e manter um estilo de vida sedentário. Os resultados do estudo podem estar relacionados com a falta de conscientização por parte dos indivíduos obesos "sem complicações" sobre os riscos da doença para sua saúde.

Nesse percurso é importante lembrar que os pacientes não são os únicos responsáveis pelo tratamento, já que, como foi explicitado anteriormente, diversos fatores interferem no processo terapêutico, contribuindo para a não adesão, como valores, crenças, aspectos relativos aos serviços de saúde e relacionamento profissional-paciente..$^{5,9,18,20}$

Estudos revelam a decepção dos pacientes obesos por ter de tratar a doença na atenção primária por falta de tempo, experiência e baixo assessoramento recebido pelos profissionais. Alguns autores sugerem que os profissionais de saúde de atenção primária mostram atitudes negativas e desinteresse no tratamento de obesos. ${ }^{4}$ Contudo, é importante ressaltar o desgaste dos profissionais devido à sobrecarga de trabalho diário e a baixa remuneração, que são fatores que influenciam esse tipo de comportamento. ${ }^{4,18}$

A adesão a um tratamento é uma mudança comportamental em busca de um estilo de vida saudável, o que inclui mudanças de hábitos alimentares que são frequentemente interpretadas como algo desagradável e difícil de realizar. ${ }^{24,39}$ Diversos fatores contribuem para que as pessoas acreditem que a dieta seja a parte mais difícil do tratamento de ser realizada, o que influencia ainda mais a baixa adesão, como planos dietéticos inflexíveis que não levam em consideração aspectos pessoais dos pacientes e a visão restritiva que os pacientes possuem dos planos alimentares. . $^{8,39}$ Nada deve ser proibido, desde que sejam respeitadas as quantidades preestabelecidas. ${ }^{1}$ Sobretudo, o padrão alimentar está envolvido com questões emocionais e culturais, por isso muitas vezes é difícil de modificar. ${ }^{39}$ É muito importante a conscientização em relação à necessidade da adesão ao tratamento dietético, mas deve-se ter atenção e respeito aos costumes e crenças dos pacientes. A intervenção nutricional será mais bem-sucedida se a proposta educativa estiver centrada nas crenças, costumes e preocupações dos pacientes sobre o tratamento. ${ }^{39,44} \mathrm{O}$ conceito de mudança de hábito alimentar está ligado intimamente à ideia de processo progressivo, que requer flexibilidade por parte dos profissionais, que ocorre de forma lenta e gradual, podendo advir retrocessos que dependem dos acontecimentos na vida dos pacientes, como perdas, problemas familiares, mudanças drásticas de rotina etc. ${ }^{8}$ 
Estratégias no atendimento nutricional que promovam motivação do indivíduo poderiam melhorar a adesão ao tratamento, assim como o empoderamento do indivíduo para que ele possa se tornar capaz de identificar barreiras para o autocontrole e assim superá-las, tornando-se apto para zelar pelo seu bem-estar. ${ }^{7,16,19} \mathrm{O}$ estabelecimento de metas realistas, suporte social e familiar, bem como o de uma aliança terapêutica que permita a participação do indivíduo na solução de problemas relacionados à alimentação, são estratégias que visam reduzir as desistências inerentes ao tratamento convencional. ${ }^{19}$

O esforço para o abandono de hábitos arraigados pode despertar sentimentos de ambivalência, e na formação dos profissionais de saúde faltam informações sobre a área da comunicação e da escuta da subjetividade do ser humano, o que facilitaria o trabalho do profissional e o entendimento do paciente às orientações. ${ }^{18,42}$ A construção de vínculo entre profissional-paciente, com uma comunicação melhor e orientações mais fáceis de serem compreendidas, tanto na consulta individualizada quanto na coletiva, é um recurso terapêutico que favorece a adesão. ${ }^{10,42} \mathrm{O}$ profissional também deve estar atento ao grau de compreensão que o paciente teve das orientações realizadas, sendo capaz de identificar possíveis distorções do que foi orientado.

A palavra dieta, por exemplo, comumente utilizada pelos profissionais, traz conotação de restrição, podendo ser substituída no momento da orientação por alimentação saudável ou plano alimentar, enfatizando que, se for o caso, a alimentação saudável proposta é aquela que deveria ser adotada por todas as pessoas de modo geral, prevenindo complicações futuras para todos. ${ }^{8}$ Nesse sentido, ressalta-se a importância do nutricionista em ações de educação nutricional, já que há dificuldades por parte de outros profissionais de saúde em realizar atividades de educação e orientação nutricional, por não terem formação adequada para essa função. ${ }^{21}$

Outra estratégia para melhorar a adesão é o trabalho multiprofissional e interdisciplinar, com uma equipe de saúde ampla, a fim de encontrar alternativas viáveis às dificuldades relatadas pelos pacientes e seus familiares. ${ }^{10,11,21}$ Uma abordagem multiprofissional permite que os pacientes tenham uma adequada compreensão de sua condição, pois os profissionais não médicos fornecem, muitas vezes, as mesmas informações de diferentes formas, facilitando o entendimento do paciente sobre a importância do tratamento da doença. ${ }^{18,27}$

A abordagem da não adesão também deve ser considerada pelos governos e instituições de ensino. Nos serviços de saúde há necessidade de educação permanente dos profissionais que atuam na rede do Sistema Único de Saúde (SUS), para desenvolver ações de prevenção e promoção de saúde como o esperado. Há necessidade também de revisão do orçamento dirigido à saúde, além de atitudes mais ativas e humanizadas em propostas de saúde pública em todos os âmbitos e em todas as esferas de governo. ${ }^{18}$ É importante ressaltar que fatores relacionados à instituição de saúde também influenciam a adesão, como o acesso ao serviço de saúde, a política de saúde e o tempo de espera de atendimento. ${ }^{1}$ 
Em diversos países, a adesão à dieta é uma meta difícil de ser alcançada. ${ }^{17}$ García-Meseguer et al., ${ }^{46} \mathrm{em}$ seu estudo transversal, caracterizaram os hábitos alimentares e avaliaram a qualidade da dieta e alguns possíveis fatores determinantes de estudantes de uma universidade espanhola, de acordo com o padrão alimentar mediterrâneo, entre outros índices. Para cada aluno (n=284) foi entregue um questionário de autorrelato envolvendo medidas antropométricas, tipos de habitação, hábitos de fumar e níveis de atividade física. O consumo alimentar foi coletado através de dois R24h não consecutivos, incluindo um dia de fim de semana. A avaliação da qualidade da dieta se deu através do Healthy Eating Index (HEI) e Escore da Dieta Mediterrânea, que revelaram baixa-intermediária pontuação em ambos. Segundo a classificação do HEI, 96,1\% dos indivíduos marcaram "pobre" ou "necessita de melhorias" sobre a qualidade de sua dieta e apenas 5,3\% dos estudantes alcançaram uma alta adesão à dieta Mediterrânea.

Numa outra direção, Torrado et al., ${ }^{47}$ em estudo longitudinal realizado em Madri com 99 adultos obesos submetidos a um programa de redução de peso, objetivaram conhecer o comportamento emocional dos indivíduos para facilitar o estabelecimento de orientações nutricionais personalizadas baseadas em hábitos alimentares saudáveis e aumentar a fidelidade do paciente até atingir o peso desejado. A adesão ao padrão alimentar Mediterrâneo e o efeito das emoções sobre as escolhas alimentares foram determinados com a aplicação dos questionários MEDAS (Mediterranean Diet Adherence Screener) e EEQ (Emocional Eater Questionnaire), respectivamente. A população estudada tinha estilo de vida pouco saudável e seu comportamento alimentar era dependente das emoções. A maioria dos participantes (66\% das mulheres e $71 \%$ dos homens) foi classificada como comedores emocionais. Durante o programa de tratamento, os participantes mudaram seus hábitos alimentares e estilo de vida, conseguindo reduzir o peso corporal em pelo menos $10 \%$ do peso inicial.

Por meio desse estudo, ressalta-se a necessidade de levar em consideração os sentimentos dos pacientes em relação aos alimentos, para que assim possa se desenvolver uma estratégia individualizada para cada indivíduo de acordo com suas possibilidades, visando vencer as barreiras que o impeçam de seguir as orientações propostas.

Um estudo $^{48}$ de coorte observacional, desenvolvido na Austrália, comprovou que alta qualidade da dieta se associa a melhor qualidade de vida e capacidade funcional em adultos mais velhos $(\geq$ 55 anos). Foram analisados 1.305 e 895 participantes com dados completos depois de cinco e dez anos, respectivamente. Os dados dietéticos foram recolhidos usando um QFA semiquantitativo autoadministrado, contendo 145 itens alimentares e nove categorias de frequência, para indicar a regularidade habitual de itens alimentares consumidos no último ano. Foram determinados escores totais da dieta para a ingestão de grupos de alimentos e nutrientes, refletindo a adesão às diretrizes dietéticas, conforme o Guia para Alimentação Saudável Australiano. Escores mais altos indicavam maior adesão às orientações nutricionais. 
Escores de dieta podem servir como ferramentas úteis para avaliar a adesão da população a uma dieta e para estudar os benefícios para a saúde de um determinado padrão alimentar, ${ }^{46} \mathrm{e}$ diversos estudos fizeram uso desses escores, como o Escore da Dieta Mediterrânea ou o Escore DASH (Dietary Approaches to Stop Hypertension). ${ }^{49-54}$ Esses índices avaliam a qualidade da dieta individual e populacional em termos de conformidade com a orientação dietética, ${ }^{46,55}$ e entre os principais destaca-se o HEI, desenvolvido de acordo com as diretrizes alimentares americanas. Alguns estudos adaptaram o HEI para a população brasileira. Os índices são compostos geralmente por grupos de alimentos e nutrientes, e para determinação dos padrões alimentares é necessário conhecer sua construção e pontuação. Podem ser utilizados também na determinação de estratégias e intervenções nutricionais mais eficientes, auxiliando programas de educação nutricional. ${ }^{55}$ Entretanto, como todo instrumento de análise alimentar, apresenta vantagens e limitações, já que representar a dimensão do comportamento alimentar humano é algo complexo, que requer não apenas a utilização de técnicas adequadas, mas habilidades interpessoais do entrevistador para que o indivíduo relate da melhor forma possível o que consome..$^{55}$

No que se refere às doenças pulmonares, a adesão terapêutica também é baixa. ${ }^{56} \mathrm{~A}$ fibrose cística (FC), por exemplo, exige a adesão do paciente a um tratamento complexo de autocuidado que preenche uma parcela considerável do seu tempo de vida. Dalcin et al. ${ }^{25}$ estudaram a adesão autorrelatada de 38 pacientes atendidos por um programa de adultos com FC, em estudo transversal, prospectivo, estabelecendo associações com as características clínicas da doença e com a percepção da adesão do paciente pela equipe multidisciplinar. O grau da adesão foi avaliado por questionário adaptado, em que foram desenvolvidas duas formas de questionários: uma dirigida aos profissionais da equipe multidisciplinar e outra dirigida ao paciente. As duas formas de questionários abordavam sete das recomendações terapêuticas principais para o manejo da doença, como fisioterapia respiratória, dieta hipercalórica e enzimas pancreáticas e, em cada questão, deveria ser assinalada a frequência semanal de utilização da modalidade terapêutica considerada. Foi evidenciada elevada adesão autorrelatada para a maioria das recomendações terapêuticas. Entretanto, duas recomendações tiveram adesão muito baixa: a dieta e a atividade física. O escore de adesão autorrelatada se correlacionou inversamente com o escore clínico, sugerindo que os pacientes mais graves aderem mais ao tratamento. E, de modo geral, a adesão autorrelatada pelo paciente foi maior que a percebida pela equipe de saúde.

A avaliação da adesão não se resume apenas em aferir o cumprimento das recomendações dos profissionais, mas também, mesmo que numa perspectiva limitada, verificar fatores que dificultam e tornam distantes da realidade de muitos pacientes a incorporação de certas atitudes necessárias à terapêutica. Sobretudo, no processo de adesão o paciente tem autonomia para aceitar ou não as recomendações, devendo ser, como explanado anteriormente, um participante ativo da trapêutica. ${ }^{8}$

Dessa forma, para criar soluções para a promoção da adesão é fundamental identificar os obstáculos que impedem a adesão, as dificuldades dos pacientes em relação ao tratamento e que 
tipos de condutas poderiam tornar possível o seguimento das recomendações. ${ }^{10,57}$ Ademais, essa identificação pode possibilitar ainda o reconhecimento dos grupos mais vulneráveis à não adesão, contribuindo para a adoção de ações voltadas a esses grupos. ${ }^{18}$

Um estudo multistate ${ }^{57}$ realizado em seis locais dos Estados Unidos, objetivou compreender barreiras e facilitadores para adesão ao Dietary Guidelines for Americans para quatro grupos de alimentos (leite, grãos integrais, frutas e hortaliças), em crianças da quinta série e cuidadores adultos. As barreiras identificadas para os cuidadores adultos foram: falta de habilidades para a preparação da refeição ou receitas, dificuldade em mudar hábitos alimentares, custo, falta de conhecimento da recomendação/porção/benefícios para a saúde e sabor. Para as crianças, as barreiras centrais foram: alimentos concorrentes (alimentos com alto teor calórico, mas com níveis reduzidos de nutrientes), preocupações com a saúde (como alergia ao leite/dor de estômago), sabor/aroma/cheiro, esquecer de comê-los e dificuldade de consumir ou descobrir a quantidade recomendada. Os autores sugerem que as intervenções poderiam se direcionar para barreiras específicas, no intuito de mudar o consumo de um determinado grupo de alimentos em vez de focar em comportamentos alimentares em geral, o que vai de encontro com o proposto por Rodríguez et al., ${ }^{24}$ de que as mudanças sejam realizadas apenas em grupos de alimentos que estão sendo consumidos de forma inadequada.

Os fatores socioeconômicos também são determinantes da falta de adesão, já que quanto mais baixos estes níveis, menores são as taxas de adesão, possivelmente pelo menor conhecimento da doença e mais difícil o acesso aos serviços de saúde. ${ }^{18}$ Entretanto, observou-se que em grande parte dos estudos analisados na presente pesquisa não foram encontradas associações entre a adesão e as características sociodemográficas ou sobre o conhecimento acerca da doença, o que pode ter sido influenciado pelo tamanho relativamente reduzido das amostras em alguns estudos, pelos variados delineamentos das pesquisas e pela diversidade de locais em que foram realizadas.

\section{Conclusão}

Há várias interfaces que podem influenciar a adesão ao tratamento, como a falta de motivação para modificação de estilo de vida, a falta de conhecimento acerca da doença, a baixa autoestima ou a depressão, a ausência de apoio familiar e social, os problemas pessoais, o elevado número de doenças associadas, a ausência de sintomas, o estresse, as representações negativas em relação à doença e ao tratamento, o relacionamento com a equipe de saúde e a instituição de saúde, a escolaridade do paciente, o sentimento de bem-estar.

A adesão às mudanças de hábitos é um processo dinâmico, sendo desafiador não só para os pacientes, mas também para os profissionais de saúde, que necessitam desenvolver estratégias que tornem possível o aumento da adesão. Proporcionar tratamentos mais flexíveis, com objetivos claros, 
com enfoque em mudanças graduais, com maior acompanhamento, com trabalho multiprofissional e interdisciplinar, pode possibilitar maior adesão ao tratamento.

Evitar prescrições muito restritivas e incompatíveis com o estilo de vida de alguns pacientes é um dos primeiros passos para melhorar a adesão às orientações nutricionais, que se relaciona ao reconhecimento, à aceitação e à adaptação da nova condição de saúde pelo indivíduo, além do desenvolvimento de autocuidado para buscar hábitos mais saudáveis. Quanto menor a complexidade, maior será a adesão terapêutica.

Com base nessa perspectiva, a existência de instrumentos validados que consigam mensurar e avaliar o comportamento do indivíduo, para além do manejo empírico de dados que se obtém pela simples observação, é importante para aplicação em pesquisas e para guiar os profissionais em sua conduta, permitindo certa precisão sobre esse dado subjetivo.

Devido à complexidade da dimensão da temática adesão não existe um método ou instrumento que se adapte a todos os objetivos e pesquisas. Contudo, são necessários estudos metodológicos que elaborem e validem instrumentos de avaliação da adesão às orientações nutricionais, além do tratamento como um todo, visto que há instrumentos que visam aferir o tratamento de uma forma geral, porém, atribuem maior ênfase ao tratamento farmacológico.

\section{Colaboradores}

Estrela KCA trabalhou em todas as etapas de elaboração do estudo, desde a concepção até a revisão da versão final do artigo; Alves ACC participou de todas as etapas de elaboração do estudo, desde a concepção até a revisão da versão final do artigo; Gomes TT participou da revisão da versão final do artigo; Isosaki M participou da redação do artigo e da sua versão final.

Conflito de Interesses: Os autores declaram não haver conflito de interesses.

\section{Referências}

1. Bueno JM, Leal FS, Saquy LPL, Santos CB, Ribeiro RPP. Educação alimentar na obesidade: adesão e resultados antropométricos. Rev Nutr 2011; 24(4):575-84.

2. Busnello FM, Bodanese LC, Pellanda LC, Santos ZEA. Intervenção nutricional e o impacto ao tratamento em pacientes com síndrome metabólica. Arq Bras Cardiol 2011; 97(3):217-24.

3. Rodrigues MTP, Moreira TMM, Andrade DF. Elaboração e validação de instrumento avaliador da adesão ao tratamento da hipertensão. Rev Saúde Públ 2014; 48(2):232-9.

4. Vivas EA, Plaza BL, Weber TK, López LB, Milla SP, Catalán AL, Gómez-Candela C. Variables predictoras de baja adherencia para el tratamiento del exceso de peso em atención primaria. Nutr Hosp 2013; 28(5):1530-5. 
5. Debussche X. Is adherence a relevant issue in the self-management education of diabetes? A mixed narrative review. Diabetes Metab Syndr Obes 2014; 7:357-67.

6. Borges JWP, Moreira TMM, Rodrigues MTP, Oliveira CJ. Utilização de questionários validados para mensurar a adesão ao tratamento da hipertensão arterial: uma revisão integrativa. Rev Esc Enferm USP 2012; 46(2):487-94.

7. Ferreira EAP, Fernandes AL. Treino em auto-observação e adesão à dieta em adulto com diabetes tipo 2. Psic: Teor e Pesq 2009; 25(4):629-36.

8. Pontieri FM, Bachion MM. Crenças de pacientes diabéticos acerca da terapia nutricional e sua influência na adesão ao tratamento. Ciênc Saúde Colet 2010; 15(1):151-160.

9. Jesus ES, Augusto MAO, Gusmão J, Mion Júnior D, Ortega K, Pierin AMG. Perfil de um grupo de hipertensos: aspectos biossociais, conhecimentos e adesão ao tratamento. Acta Paul Enferm 2008; 21(1):59-65.

10. Figueiredo NN, Asakura L. Adesão ao tratamento anti-hipertensivo: dificuldades relatadas por indivíduos hipertensos. Acta Paul Enferm 2010; 23(6):782-7.

11. Costa JA, Balga RSM, Alfenas RCG, Cotta RMM. Promoção da saúde e diabetes: discutindo a adesão e a motivação de indivíduos diabéticos participantes de programas de saúde. Ciênc Saúde Colet 2011; 16(3):2001-9.

12. Chow CK, Jolly S, Rao-Melacini P, Fox KAA, Anand SS, Yusuf S. Association of diet, exercise, and smoking modification with risk of early cardiovascular events after acute coronary syndromes. Circulation 2010; 121:750-8.

13. Davison KAK, Negrato CA, Cobas R, Matheus A, Tannus L, Plama CS, et al. Relationship between adherence to diet, glycemic control and cardiovascular risk factors in patients with type 1 diabetes: a nationwide survey in Brazil. Nutr J 2014; 13:19.

14. Schultz RDP, Wichmann FA, Couto AN. Adesão e eficácia do aconselhamento dietético após intervenção nutricional em mulheres com fatores de risco para a síndrome metabólica. Cinergis 2014; 15(3):123-8.

15. Girotto E, Andrade SM, Cabrera MA, Matsuo T. Adesão ao tratamento farmacológico e não farmacológico e fatores associados na atenção primária da hipertensão arterial. Ciênc Saúde Colet 2013; 18(6):1763-72.

16. Silva LS, Cotta RMM, Ribeiro AQ, Ribeiro AG. A problemática da adesão ao tratamento da hipertensão no contexto da Saúde da Família. Mundo Saúde 2014; 38(4):375-83.

17. Campuzano MRL, Rodríguez AR, Rodríguez JCG. Adherencia a la dieta en pacientes diabéticos: efectos de una intervención. Summa psicol UST 2013; 10(1):91-101.

18. Lustosa MA, Alcaires J, Costa JC. Adesão do paciente ao tratamento no Hospital Geral. Rev SBPH 2011; 14(2):27-49.

19. Guimarães NG, Dutra ES, Ito MK, Carvalho KMB. Adesão a um programa de aconselhamento nutricional para adultos com excesso de peso e comorbidades. Rev Nutr 2010; 23(3):323-33. 
20. Borges JWP, Moreira TMM, Rodrigues MTP, Souza ACC, Silva DB. Validação de conteúdo das dimensões constitutivas da não adesão ao tratamento da hipertensão arterial. Rev Esc Enferm USP 2013; 47(5):1077-83.

21. Ribeiro AG, Cotta RMM, Silva LS, Ribeiro SMR, Dias CMGC, Mitre SM, et al. Hipertensão arterial e orientação domiciliar: o papel estratégico da saúde da família. Rev Nutr 2012; 25(2):271-82.

22. Tarasautchi D, Coelho LC, Silva CVD, Sachs A, Asakura L. Avaliação da intervenção nutricional em pacientes com síndrome metabólica. Rev Bras Nutr Clin 2010; 25(3):189-94.

23. Oliveira A, Vieira LP, Hinnig PF. Triagem e avaliação nutricional. In: Vieira LP, Isosaki M, Oliveira A, Costa HM. Terapia nutricional em cardiologia e pneumologia: com estudos de casos comentados. São Paulo: Atheneu; 2014. p.3-209.

24. Rodríguez PC, Guerrero DB, Díaz SP. Elaboración y validación de un nuevo cuestionario de hábitos alimentarios para pacientes con sobrepeso y obesidade. Endocrinol Nutr 2010; 57(4):130-9.

25. Dalcin PTR, Rampon G, Pasin LR, Ramon GM, Abrahão CLO, Oliveira VZ. Adesão ao tratamento em pacientes com fibrose cística. J Bras Pneumol 2007; 33(6):663-70.

26. Saccomann ICRS, Cintra FA, Gallani MCBJ. Fatores associados às crenças sobre adesão ao tratamento não medicamentoso de pacientes com insuficiência cardíaca. Rev Esc Enferm USP 2014; 48(1):18-24.

27. Alves FD, Souza GC, Brunetto S, Perry IDS, Biolo A. Nutritional orientation, knowledge and quality of diet in heart failure; randomized clinical trial. Nutr Hosp 2012; 27(2):441-8.

28. Mussi CM, Ruschel K, Souza EN, Lopes ANM, Trojahn MM, Paraboni CC, et al. Home visit improves knowledge, self-care and adhesion in heart failure: randomized Clinical Trial HELEN-I. Rev Latino-Am Enfermagem 2013; 21(spe number):20-8.

29. Silva AF, Cavalcanti ACD, Malta M, Arruda CS, Gandin T, Fé A, et al. Treatment adherence in heart failure patients followed up by nurses in two specialized clinics. Rev Latino-Am Enfermagem 2015; 23(5):888-94.

30. Mantovani VM, Ruschel KB, Souza EN, Mussi C, Rabelo-Silva ER. Adesão ao tratamento de pacientes com insuficiência cardíaca em acompanhamento domiciliar por enfermeiros. Acta Paul Enferm 2015; 28(1):41-7.

31. Rabelo ER, Mantovani VM, Aliti GB, Domingues FB. Cross-cultural adaptation and validation of a disease knowledge and self-care questionnaire for a brazilian sample of heart failure patients. Rev Latino-Am Enfermagem 2011; 19(2):277-84.

32. Feijó MK, Ávila CW, Souza EN, Jaarsma T, Rabelo ER. Cross-cultural adaptation and validation of the European Heart Failure Self-care Behavior Scale for Brazilian Portuguese. Rev Latino-Am Enfermagem 2012; 20(5):988-96.

33. d'Almeida KS, Souza GC, Rabelo-Silva ER. Adaptação transcultural para o Brasil do Dietary Sodium Restriction Questionnaire (Questionário de Restrição de Sódio na Dieta) (DSRQ). Arq Bras Cardiol 2012; 98(1):70-5.

34. d'Almeida KS, Souza GC, Rabelo-Silva ER. Validity and reliability of the Dietary Sodium Restriction Questionnaire (DSRQ). Nutr Hosp 2013; 28(5):1702-09. 
35. Patton SR. Adherence to diet in youth with type 1 diabetes. J Am Diet Assoc 2011; 111:550-5.

36. Saffi MAL, Macedo Junior LJJ, Trojahn MM, Polanczyk CA, Rabelo-Silva ER. Validity and reliability of a questionnaire on knowledge of cardiovascular risk factors for use in Brazil. Rev Esc Enferm USP 2013; 47(5):1083-9.

37. Ribeiro AG, Ribeiro SMR, Dias CMGC, Ribeiro AQ, Castro FAF, Suárez-Varela MM, et al. Nonpharmacological treatment of hypertension in primary health care: A comparative clinical trial of two education strategies in health and nutrition. BMC Public Health 2011; 11:637.

38. Racine E, Troyer JL, Warren-Findlow J, Mcauley WJ. The effect of medical nutrition therapy on changes in dietary knowledge and dash diet adherence in older adults with cardiovascular disease. J Nutr Health Aging 2011; 15(10):868-76.

39. Nascimento NC, Zaparolli MR, Baptista DR, Vayego SA. Adesão à terapia nutricional por pacientes diabéticos internados em um hospital público do município de Curitiba-PR. Rev Atenção Saúde 2014; 12(41):5-10.

40. Assunção TS, Ursine PGS. Estudo de fatores associados à adesão ao tratamento não farmacológico em portadores de diabetes mellitus assistidos pelo Programa Saúde da Família, Ventosa, Belo Horizonte. Ciênc Saúde Colet 2008; 13(Suppl 2):2189-97.

41. Michels MJ, Coral MHC, Sakae TM, TB Damas, Furlanetto LM. Questionário de Atividades de Autocuidado com o Diabetes: tradução, adaptação e avaliação das propriedades psicométricas. Arq Bras Endocrinol Metab 2010; 54(7):644-51.

42. Franzoni B, Lima LA, Castoldi L, Labrea MGA. Avaliação da efetividade na mudança de hábitos com intervenção nutricional em grupo. Ciênc Saúde Colet 2013; 18(12):3751-8.

43. Veiga DKE, Santos EO, Souza MKT, Leite SKL, Liberali R, Coutinho VF. Avaliação dos estágios motivacionais de indivíduos que procuram atendimento nutricional. Encon: R Psicol 2010; 13(18):117-25.

44. Martins AG, Chavaglia SR, Ohl RI, Martins IM, Gamba MA. Adesão ao tratamento clínico ambulatorial da hipertensão arterial sistêmica. Acta Paul Enferm 2014; 27(3):266-72.

45. Moreira P, Romualdo MCS, Amparo FC, Paiva C, Alves R, Magnoni D, et al. A educação nutricional em grupo e sua efetividade no tratamento de pacientes obesos. Rev Bras Obes Nutr Emagrecimento 2012; 6(35):216-24.

46. García-Meseguer MJ, Burriel FC, García CV, Serrano-Urrea R. Adherence to Mediterranean diet in a Spanish university population. Appetite 2014; 78:156-64.

47. Torrado YP, Velasco AG, Galiot AH, Cambrodón IG. A strategy for weight loss based on healthy dietary habits and control of emotional response to food. Nutr Hosp 2015; 31(6):2392-9.

48. Gopinath B, Russell J, Flood VM, Burlutsky G, Mitchell P. Adherence to dietary guidelines positively affects quality of life and functional status of older adults. J Acad Nutr Diet 2014; 114:220-9.

49. Ozen AE, Bibiloni Mdel M, Murcia MA, Pons A, Tur JA. Adherence to the Mediterranean diet and consumption of functional foods among the Balearic Islands' adolescent population. Public Health Nutr 2015; 18(4):659-68. 
50. Fung TT, Chiuve SE, McCullough ML, Rexrode KM, Logroscino G, Hu FB. Adherence to a DASHstyle diet and risk of coronary heart disease and stroke in women. Arch Intern Med 2008; 168(7):713-20.

51. Reedy J, Krebs-Smith SM, Miller PE, Liese AD, Kahle LL, Park Y, Subar AF. Higher diet quality is associated with decreased risk of all-cause, cardiovascular disease, and cancer mortality among older adults. J Nutr 2014;144(6):881-9.

52. Kröger J, Schulze MB, Romaguera D, Guevara M, Buijsse B, Boeing H, et al. Adherence to predefined dietary patterns and incident type 2 diabetes in European populations: EPIC-InterAct Study. Diabetologia 2014; 57:321-33.

53. Bonaccio M, Di Castelnuovo A, De Curtis A, Costanzo S, Persichillo M, Donati MB, et al. Adherence to the Mediterranean diet is associated with lower platelet and leukocyte counts: results from the Moli-sani study. Blood 2014; 123(19):3037-44.

54. Atkins JL, Whincup PH, Morris RW, Lennon LT, Papacosta O, Wannamethee SG. High diet quality is associated with a lower risk of cardiovascular disease and all-cause mortality in older men. J Nutr 2014; 144(5):673-80.

55. Previato HDRA, Volp ACP, Freitas RN. Avaliação da qualidade da dieta pelo Índice de Alimentação Saudável e suas variações: uma revisão bibliográfica. Nutr Clín Diet Hosp 2014; 34(2):88-96.

56. Leiva-Fernández J, Leiva-Fernández F, García-Ruiz A, Prados-Torres D, Barnestein-Fonseca P. Efficacy of a multifactorial intervention on therapeutic adherence in patients with chronic obstructive pulmonary disease (COPD): a randomized controlled trial. BMC Pulm Med 2014; 14:70.

57. Nicklas TA, Jahns L, Bogle ML, Chester DN, Giovanni M, Klurfeld DM, Laugero K, Liu Y, Lopez S, Tucker KL. Barriers and facilitators for consumer adherence to the dietary guidelines for Americans: the HEALTH study. J Acad Nutr Diet 2013; 113:1317-31.

Recebido: 10/4/2016

Revisado: 25/10/2016

Aceito: $29 / 1 / 2017$ 